\title{
AC 2012-3299: AN EXPERIMENT TO INTRODUCE PH-RESPONSIVE HYDROGELS FOR CONTROLLED DRUG DELIVERY
}

\section{Dr. Stephanie Farrell, Rowan University}

Stephanie Farrell is an Associate Professor in chemical engineering at Rowan University. Prior to joining Rowan in 1998, she was an Assistant Professor in chemical engineering and Adjunct Professor in biomedical engineering at Louisiana Tech University. She received her bachelor's, M.S., and Ph.D. degrees in chemical engineering from the University of Pennsylvania, Stevens Institute of Technology, and New Jersey Institute of Technology, respectively. Farrell's educational interests are in laboratory development and experiential learning, particularly in the areas of biomedical and sustainable engineering.

\section{Prof. Jennifer Vernengo, Rowan University}

Jennifer Vernengo is an Assistant Professor of chemical engineering at Rowan University. Jennifer received her Ph.D. from Drexel University in 2007. She began work as a materials scientist at Synthes Biomaterials, then joined Drexel University College of Medicine as postdoc in 2009. Vernengo's research is in the area of injectable biomaterials for orthopedic tissue replacement and repair. She is particularly interested in developing innovative approaches to biomedical engineering education.

\section{Stephen E. Montgomery \\ Yang Zhang, Rowan University \\ Mr. Peter John Schwalbenberg, Rowan University}

Peter Schwalbenberg is studying chemical engineering at Rowan University, specializing in materials for his bachelor of science degree (anticipated graduation date May 2013). He has been employed as a Laboratory Assistant for the processing technician of the Chemical Engineering Department at Rowan University. He has also researched polydimethyl-siloxane (PDMS) micro bubbles for the David Weitz laboratory at Harvard University and has presented his research at the American Institute of Chemical Engineers (AIChE) National Student Conference. Peter currently works as a Resident Assistant (RA) on campus, as well as being a full-time student. 


\section{An Experiment to Introduce pH-responsive Hydrogels for Controlled Drug Delivery}




\section{Abstract}

Stimuli-responsive polymers are used in a variety of biomedical applications. For example, $\mathrm{pH}$ responsive hydrogels have been extensively investigated for controlled drug delivery. By responding to the $\mathrm{pH}$ environment in the body, which changes depending on location and metabolic state, a $\mathrm{pH}$-sensitive drug dosage form is able to modulate drug delivery patterns to meet physiologic requirements and minimize side effects. This paper describes an experiment used to introduce freshmen engineering students to stimuli-responsive polymers for controlled release applications. Students produce a $\mathrm{pH}$ responsive hydrogel, made from polyethylene glycol grafted onto a polymethacrylic acid backbone p(MMA-EG) using free radical polymerization. These hydrogels were previously examined for oral delivery of insulin for diabetics by Nakamura et $\mathrm{al}^{1}$. In our experiment, the swelling capabilities of the hydrogels in different $\mathrm{pH}$ environments are examined as a function of crosslink density. In future experiments, hydrogel mechanical properties and release properties, as a function of these variables, will be examined. In addition to learning about $\mathrm{pH}$-responsive drug delivery, students will learn concepts of polymer chemistry, materials science, design of experiments, data analysis, and engineering design. An assessment plan will measure student mastery of learning outcomes specific to the field of biomaterials science and those set forth by ABET for undergraduate chemical engineering programs.

Keywords: $\mathrm{pH}$ Responsive Hydrogels, Oral Insulin Delivery, Diabetes, Controlled Drug Delivery

\section{INTRODUCTION}

Diabetes is a disease which affects millions of people around the world. It is classified into two major types. Type 1 Diabetes is an auto-immune disease in which, insulin-producing beta-cells within the pancreas are destroyed, resulting in insufficient insulin production by this organ. With type 2 diabetes, the body has developed a rejection to insulin and that glucose uptake cannot be regulated within a patient's muscle and fat cells. When glucose uptake cannot be regulated naturally, blood sugar levels can rise or fall to unacceptable levels and can lead to a lifethreatening diabetic coma if not carefully monitored and treated with regular insulin doses.

Diabetes is a self-managed disease, and patients must administer insulin periodically by injection to maintain blood glucose levels. Injections are painful and inconvenient, and patient non-compliance is a serious concern. The mean rate of insulin usage has been reported to be $77.44 \%$ of the prescribed dose in patients with Type I diabetes who use periodic bolus injections $^{2}$. A convenient and painless oral dosage form could help increase patient compliance, however, insulin will be degraded by the acidic $\mathrm{pH}$ of the stomach before absorption into the blood stream. For this reason, researchers have focused on the development of $\mathrm{pH}$ responsive hydrogel drug delivery systems for oral delivery of insulin and other drugs $3,4,5$.

Hydrogels are extremely hydrophilic crosslinked polymer networks that can absorb large amounts of water. They are largely used in many biomedical applications such as contact lenses, and the physical properties of hydrogels are very similar to living tissues in comparison to other synthetic biomaterials due to their high water content and rubbery properties. Hydrogels have been widely used for drug delivery systems since they allow molecules of 
different sizes to diffuse into or out of the network for drug loading and release, respectively. Since the polymer chains of different hydrogels contain specific functional groups, hydrogels can be sensitive to changes in the surrounding environment, such as the changes in $\mathrm{pH}$, temperature, and pressures. A successful $\mathrm{pH}$-responsive hydrogel for insulin delivery would exhibit very little swelling in the low $\mathrm{pH}$ of the stomach, thereby restricting the release of the drug. In the higher$\mathrm{pH}$ environment of the stomach, the hydrogel would swell, and the relaxation of the gel network structure would allow release of the drug by diffusion. Furthermore, mechanical properties of hydrogels can be modified by making relatively simple changes to the polymer structure, such as the crosslinked density.

Nakamura et al. reported a hydrogel made with polyethylene glycol chains grafted onto a polymethacrylic acid backbone $\mathrm{p}(\mathrm{MMA}-\mathrm{EG})$ that can be used to transport insulin through the stomach and into the near neutral $\mathrm{pH}$ environment of the intestines, where it can be effectively absorbed into the bloodstream ${ }^{1}$. While this hydrogel has $\mathrm{pH}$-responsive properties, there are other properties that need to be considered as well. The hydrogels must have the mechanical integrity necessary to make it through the stomach and reach the intestines intact. If the integrity of the gel structure is not maintained during transport through the GI tract, then some insulin could be released in the stomach. In the intestine, the hydrogel must release the drug gradually to avoid a burst of drug in the upper small intestine, which could result in decreased bioavailability by degradation.

Chemical engineers contribute to the design of controlled drug delivery systems which deliver a drug at a desired rate to a desired location in the body. Here, we describe an experiment in which students prepare $\mathrm{pH}$-responsive hydrogels based on $\mathrm{p}$ (MMA-EG) based on the experiments outlined by Nakamura et al. ${ }^{1}$ The properties of the hydrogels, such as swelling ratio, mechanical properties, and release characteristics are characterized as a function of $\mathrm{pH}$ and crosslink density. Students performing this experiment have the opportunity to vary the hydrogel structure in attempt to optimize the hydrogel for oral insulin delivery. Through this experiment, students will gain hands-on experience in an environment that mimics an undergraduate research experience. They will practice identifying important design variables, in this case, for drug delivery. The students will also practice translating quantitative laboratory measurements into data that can be used to evaluate a design. Lastly, they will learn aspects of polymer design and characterization, which is translatable to other areas of material science and engineering.

\section{EXPERIMENT}

In this experiment, students produce $\mathrm{pH}$ sensitive hydrogels by photo-polymerization of a monomer solution containing the photo-initiator dimethoxy propyl acetophenone, the crosslinker tetraeythylene glycol dimethacrylate, and monomers methacrylic acid, and poly(ethylene glycol) (n) monomethyl ether monomethacrylate, as described by Nakamura et alError! Bookmark not defined..

\section{Hydrogel Synthesis}

Materials

- Dimethoxy propyl acetophenone (DMPA)

- Methacrylic Acid (MAA)

- Tetraeythylene glycol dimethacrylate (TEGDMA) 
- Poly(ethylene glycol) (n) monomethyl ether monomethacrylate (PEGMA)

- Ethanol (50wt\%)

- Plain Glass Microslides (75x50 mm)

- Pasteur Pipette

- Micropipettes

- $50 \mathrm{~mL}$ Erlenmeyer flask

- Teflon sheet (0.8 mm thick)

- Small Binder Clips

- 365-nm ultraviolet light (Blak Ray, Upland, CA)

\section{Procedure}

1. In an Erlenmeyer flask, MAA, TEGMA, DMPA, PEGMA, ethanol were added in varying amounts described in Table 1. Compositions were chosen based on those proposed by Nakamura et al $^{1}$, but were modified to produce the hydrogels with the highest degree of mechanical integrity. The crosslink density of the hydrogels was modulated by varying the TEG crosslinker amount in the reaction mixture from low to high $(1.04 \mathrm{~mL}$ to $1.56 \mathrm{~mL}$ to $2.08 \mathrm{~mL})$ in order to investigate the effects of hydrogel properties.

Table 1. Compositions of hydrogels made and their durability in pH 7.4

\begin{tabular}{ccccc}
\hline $\begin{array}{c}\text { Mass of } \\
\text { PEGMA }(\mathrm{g})\end{array}$ & $\begin{array}{c}\text { Mass of } \\
\text { MAA }(\mathrm{mL})\end{array}$ & $\begin{array}{c}\text { Mass of } \\
\text { TEGDMA }(\mathrm{mL})\end{array}$ & $\begin{array}{c}\text { Mass of } \\
\text { DMPA }(\mathrm{g})\end{array}$ & $\begin{array}{c}\text { Mass of } \\
\text { EtOH }(\mathrm{g})\end{array}$ \\
\hline 1 & 1.9233 & 1.04 & 0.0204 & 4.0881 \\
1 & 1.9233 & 1.56 & 0.0233 & 5.0950 \\
1 & 1.9233 & 2.08 & 0.0261 & 5.7163 \\
\hline
\end{tabular}

2. The solution was mixed well, ensuring that all of the solid particles dissolved.

3. Strips of the Teflon sheet were cut and used to keep the two microscope slides spaced $0.8 \mathrm{~mm}$ apart.

4. Binder clips were used to hold the microscope slides together.

5. Using the Pasteur pipette, the monomer solution was transferred between the microscope slides to completely fill the space between slides.

6. The microscope slides with solution were then placed underneath the UV lamp and the lamp was turned on.

7. The solution was left underneath the UV lamp for 30 minutes.

8. The UV lamp was turned off, and the hydrogel was removed from the microscope slides.

9. The hydrogel was placed in DI water for 24 hours to rinse off any possible residual monomer solution that did not polymerize.

\section{Swelling Study}

Materials

- Synthesized hydrogels

- Phosphate buffered saline (PBS) solution, $\mathrm{pH} 2.2,5.0$ or 7.4.

- 3-50mL containers 
- Stopwatch

\section{Procedure}

1. Hydrogels were broken into approximately $1 \times 1$ " pieces and dried under vacuum.

2. The dry mass was recorded.

3. Each piece was placed into a separate $50 \mathrm{~mL}$ container containing PBS solution.

4. The wet mass was recorded every 10 minutes for the first hour, then at 2, 4 and 24 hours.

\section{Experimental Calculations}

The mass swelling ratio (MSR) of the hydrogels at any time point can be calculated by using Equation 1.

where MSR is mass swelling ratio, $\mathrm{M}_{\mathrm{g}}$ is mass of gel in air, and $\mathrm{M}_{\mathrm{d}}=$ initial mass of the dried gel in air. Values for MSR as a function of time, TEGMA concentration, and $\mathrm{pH}$ were compared statistically with a student's t-test and a 95\% confidence interval to determine significance.

\section{RESULTS AND DISCUSSION}

Figure 1 shows the mass swelling ration (MSR) as a function of $\mathrm{pH}$ for the hydrogels prepared with $2.08 \mathrm{~mL}$ of TEGDMA in each $\mathrm{pH}$ environment. After 24 hours, the hydrogels in the $\mathrm{pH}$ of 7.4 had higher MSR than those in $\mathrm{pH} 2.2$ and $5.0(\mathrm{p}<0.05)$. Similar results were seen for the TEGMA concentrations.

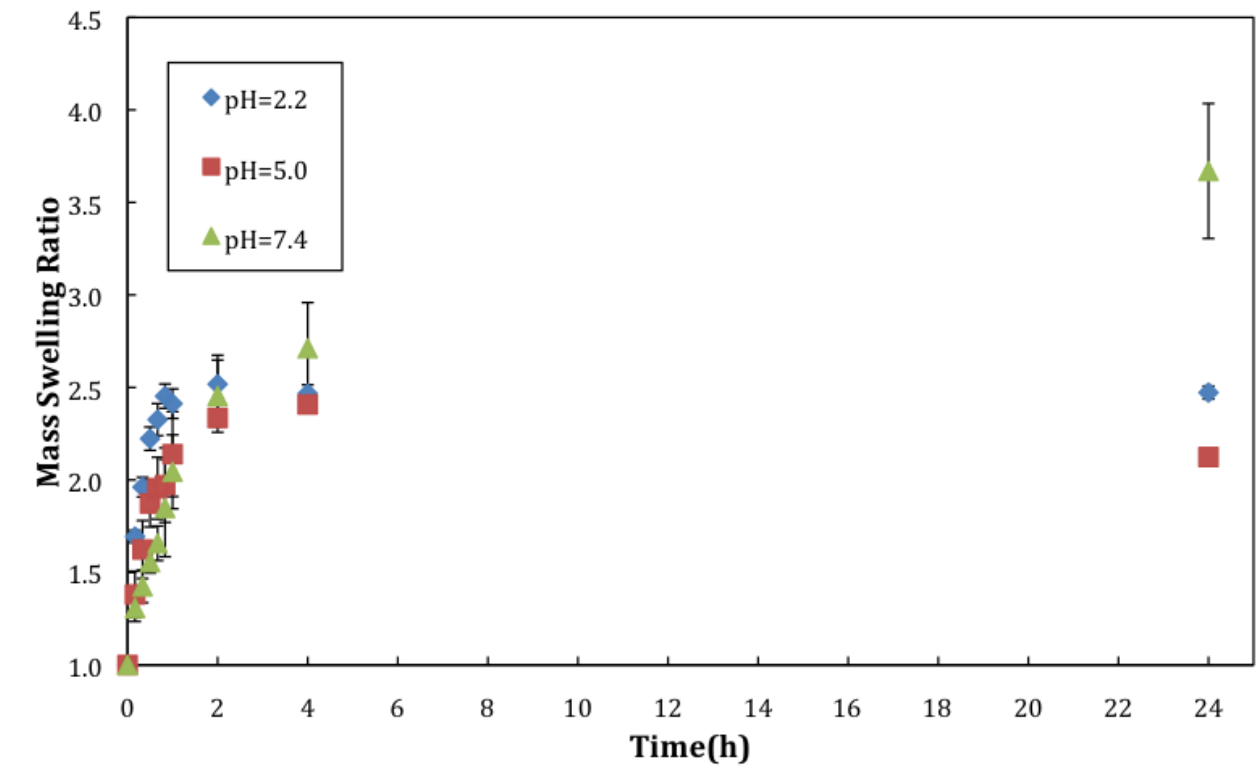

Figure 1. MSR vs. Time for $2.08 \mathrm{~mL}$ TEGDMA formulation in various pH environments. The error bars are plus or minus one standard deviation for each set.

This result is consistent with what was observed previously by researchers who characterized this system. At low $\mathrm{pH}$ values, interpolymer complexes form between the carboxylic acid protons on the PMMA and oxygen on the PEG chains. At higher $\mathrm{pH}$ values, the acid groups on the PMMA ionize, causing the polymer complexes to dissociate due to ionic 
repulsions, allowing the network to accommodate more water ${ }^{6}$. At 24 hours, hydrogels in a pH of 2.2 had an average MSR of 2.27, hydrogels in a pH of 5.0 had an average MSR of 2.32, and hydrogels placed into a $\mathrm{pH}$ of 7.4 had an average MSR of 3.95.

MSR vs. time as a function of TEGMA concentration is shown in Figure 2 at the neutral $\mathrm{pH}$ of 7.4. It was expected that increasing TEGMA concentration, or increasing crosslink density, would decrease the amount of water that can be accomodated in polymer network at equilibrium. ${ }^{7}$ However, for each $\mathrm{pH}$, no statistical differences $(\mathrm{p}<0.05)$ were seen in MSR for the hydrogels at any time point. In future work, a broader range of of crosslinker concentrations will be studied.

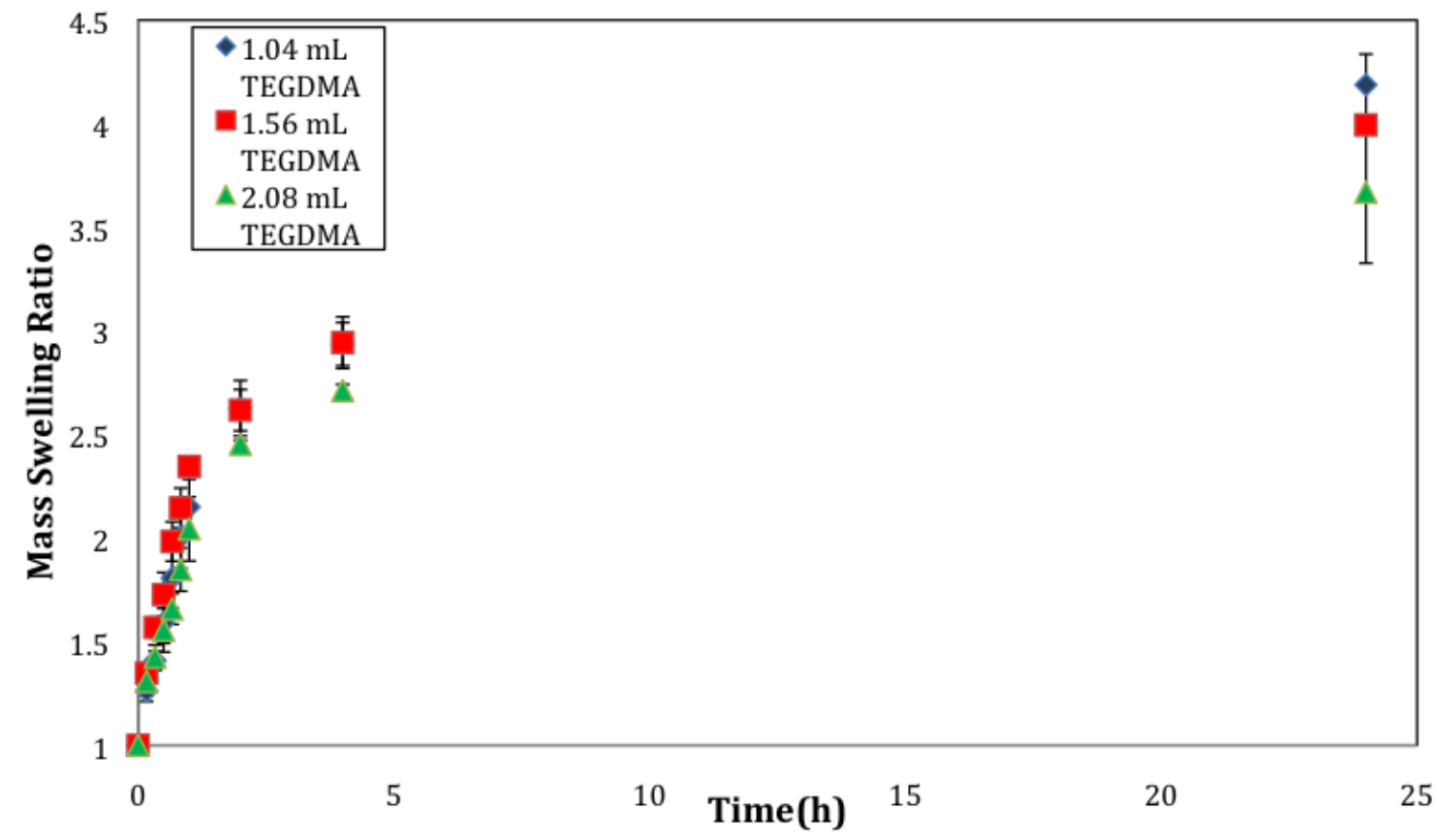

Figure 2. MSR vs. time for each hydrogel composition at $\mathrm{pH}=7.4$

\section{SUMMARY AND RECOMMENDATIONS}

In this paper, we present a laboratory activity on biomaterials and drug delivery for undergraduate engineering students. This aspect of our project focused on structure-property relationships in $\mathrm{pH}$ sensitive hydrogels for oral insulin delivery. In upcoming work, the activity will be expanded to include rubber elasticity experiments for the calculation of network mesh size as a function of $\mathrm{pH}$ and TEGMA concentration. In addition, drug release and mechanical properties will be evaluated as a function of these variables. Through this hands-on activity, students will not only develop skills specific to drug delivery and biomaterials, but in data acquisition and analysis and engineering design. The laboratory will be implemented into a freshman-level laboratory during Spring 2012. At this time, pre and post-tests will be used to gauge student mastery of learning outcomes specific to the field of biomaterials science and those set forth by ABET for undergraduate chemical engineering programs. 


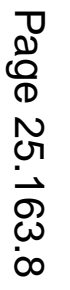




\section{References:}

${ }^{1}$ Koji Nakamura, Robert J. Murray, Jeffrey I. Joseph, Nicholas A. Peppas, Mariko Morishita, Anthony M. Lowman. Oral insulin delivery using P(MAA-g-EG) hydrogels: effects of network morphology on insulin delivery characteristics. J. Control. Release, Vol. 95, Issue 3, (2004). pp. 589-599

${ }^{2}$ Cramer, J.A., A systematic review of adherence with medications for diabetes, Diabetes Care, Vol 27, Issue 5, (2004), pp. 1218-1224.

${ }^{3}$ Piyush Gupta, Kavita Vermani, Sanjay Garg. Hydrogels: from controlled release to $\mathrm{pH}$-responsive drug delivery. Drug Discovery Today, Vol. 7, Issue 10,(2002), pp 569-579.

${ }^{4}$ L Ferreira, et al. Evaluation of poly(2-hydroxyethyl methacrylate) gels as drug delivery systems at different $\mathrm{pH}$ values. Int. J. Pharm.194 (2000), pp. 169-180.

${ }^{5}$ A Bilia, et al. In vitro evaluation of a pH-sensitive hydrogel for control of GI drug delivery from silicone-based matrices. Int. J. Pharm. 130 (1996), pp. 83-92.

${ }^{6}$ A.M. Lowman, N.A. Peppas, Analysis of the complexation/decomplexation phenomena in polyelectolyte networks, Macromelecules, 30 (1997) 4659- 4965

${ }^{7}$ Ratner B, Hoffman AS, Schoen FJ, Lemons JE. Biomaterials Science: A Multidisciplinary Endeavor. Biomaterials Science: A Introduction to Materials in Medicine. San Diego: Elsevier Academic Press; 2004. p. 1-9. 